\title{
TROU D'OZONE ET PRIX NOBEL 1995 DE CHIMIE
}

\author{
André Berger \\ Université catholique de Louvain \\ Institut d'astronomie et de géophysique Georges-Lemaître \\ 2, chemin du Cyclotron \\ 1348 Louvain-la-Neuve \\ BELGIQUE
}

RÉSUMÉ À l'occasion de la remise du prix Nobel 1995 de chimie à trois spécialistes mondiaux de la chimie de l'atmosphère, cet article retrace l'historique des connaissances sur la chimie de l'ozone stratosphérique. Il résume ensuite ce que l'on sait actuellement de l'altération de la couche d'ozone et de son impact sur le climat, la végétation et la santé des hommes.

\section{ABSTRACT The ozone hole and the 1995 Nobel Prize in chemistry}

To mark to award of the 1995 Nobel Prize in chemistry to three world renowned atmospheric chemists, this paper recalls the history of scientific progress in stratospheric ozone chemistry. Then it summarizes current knowledge of ozone-layer depletion and its impact on climate, vegetation and human health.

\section{LES PRIX NOBEL 1995}

L'ozone, molécule triatomique d'oxygène, est un composant mineur de l'atmosphère terrestre. Sa concentration varie dans l'espace et dans le temps, en particulier sous l'action des activités humaines.

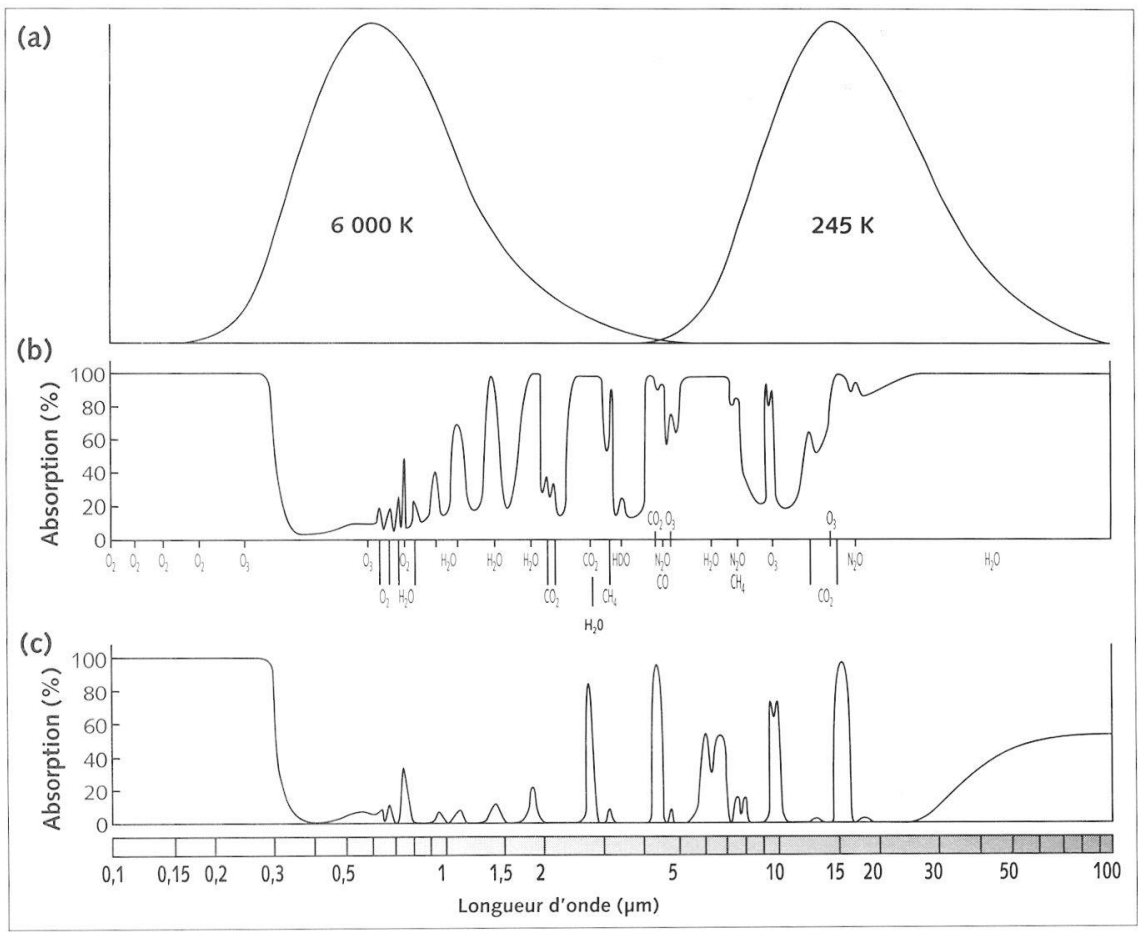

Bien que sa concentration soit faible (au maximum 10 molécules pour un million de molécules d'air), l'ozone joue un rôle exceptionnel dans le maintien de la vie sur Terre. Avec l'oxygène moléculaire, il absorbe la majeure partie du rayonnement ultraviolet qui nous vient du Soleil (figure 1) et protège ainsi le monde vivant de ces rayons dangereux. Sans la couche protectrice d'ozone, plantes et animaux ne pourraient résister, du moins en surface. Il est dès lors fondamental de comprendre les processus qui régissent la concentration d'ozone dans l'air.

Figure 1 - (a) Émission du corps noir à 6000 $\mathrm{K}$ (Soleil) et $245 \mathrm{~K}$ (Terre) ; spectre d'absorption du rayonnement solaire au sol (b) et à la tropopause (c). Conditions typiques de nos latitudes moyennes (tiré de Meteorological Glossary, Met. Office, Londres, 1991, p. 2). Crown copyright is reproduced with the permission of the controller of HMSO. 
Paul Crutzen, Mario Molina et Sherwood Rowland ont reçu le prix Nobel de chimie 1995 pour leurs auvres pionnières qui ont permis de comprendre comment l'ozone est formé et détruit par diverses réactions chimiques dans l'atmosphère. Plus spécifiquement, leurs travaux ont montré la sensibilité de la couche d'ozone à l'influence des émissions de certains produits directement liés aux activités humaines. Ils ont donc contribué de manière exceptionnelle à donner les informations scientifiques indispensables pour permettre à la société d'éviter les conséquences désastreuses qui pourraient accompagner tout changement profond de notre environnement planétaire.

Paul Crutzen est né en 1933 à Amsterdam. Docteur ès sciences météorologiques de l'université de Stockholm en 1973, il est directeur de l'Institut Max Planck de chimie à Mayence. Il est membre de l'Académie

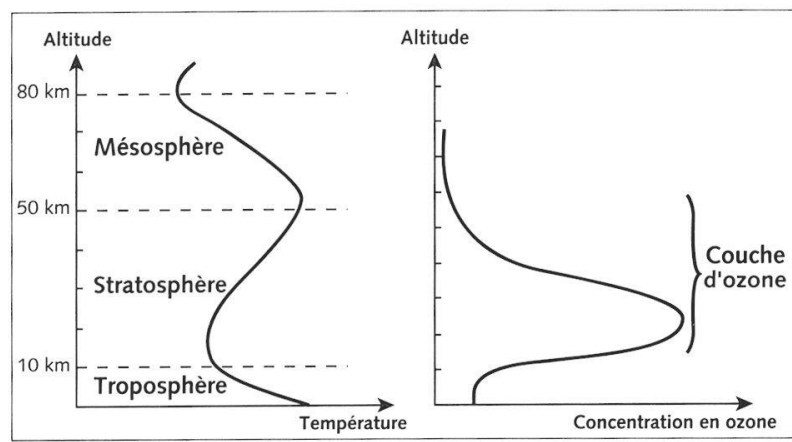

Figure 2 - Profils verticaux typiques de température (la troposphère et la stratosphère) et d'ozone (tiré de Berger, 1992). royale suédoise des sciences, de l'Académie royale suédoise des sciences de l'ingénieur et de l'Academia Europaea. Il est Docteur honoris causa de la faculté des sciences de l'Université catholique de Louvain (1992).

Mario Molina est né en 1943 à Mexico. Il est titulaire d'un Ph.D. en chimie physique de l'université de Californie à Berkeley et travaille au Massachusetts Institute of Technology de Cambridge (États-Unis). Il est membre de la National Academy of Sciences des États-Unis.

F. Sherwood Rowland est né en 1927 à Delaware (Ohio, États-Unis). Il est docteur ès sciences chimiques de l'université de Chicago (1952) et travaille à l'université de Californie à Irvine (Californie, États-Unis). Il est Foreign Secretary de la National Academy of Sciences des États-Unis.

L'OZONE

On dénombre actuellement quelque 3000 composants chimiques dans l'atmosphère terrestre. Parmi eux, 170 sont réactifs dans la troposphère. Bien que la stratosphère soit chimiquement moins complexe que la troposphère, on n'y dénombre pas moins de 40 espèces chimiques importantes. La concentration en ozone est maximale entre 15 et $50 \mathrm{~km}$, c'est-à-dire au sein de cette stratosphère (figure 2) qui renferme environ $90 \%$ de tout l'ozone. Le maximum est de l'ordre de 5 ppmv $^{(1)}$, mais, dans cette couche, la concentration varie de 0,2 à 10 ppmv environ. Dans la basse troposphère, la concentration est nettement plus faible et variable dans l'espace et le temps à cause de la pollution. Sa concentration en milieu industrialisé est typiquement de quelques dizaines de ppbv ${ }^{(1)}(10-200$ ppbv). En 1973, Paul Crutzen montra que l'oxydation du méthane et du monoxyde de carbone était, dans la troposphère, à l'origine de la formation d'ozone et cela, en présence de $\mathrm{NO}$ et $\mathrm{NO}_{2}$ comme catalyseurs.

Des valeurs typiques de la concentration totale intégrée le long de la verticale dans l'atmosphère sont de 300 unités Dobson (figure 3). Bien que la formation d'ozone soit maximale dans les tropiques à une

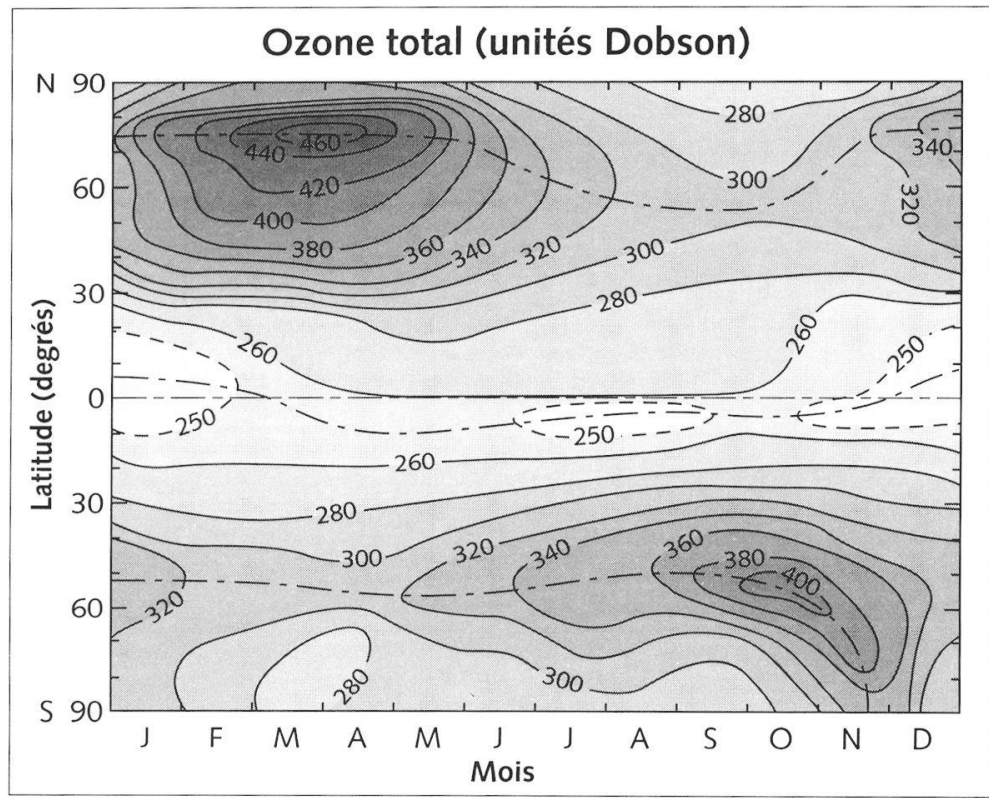
altitude d'environ 25 à $30 \mathrm{~km}$, on y observe une concentration minimale. La hauteur de la tropopause dans ces régions (de 6 à $9 \mathrm{~km}$ supérieure à celle des régions polaires) explique le transport d'ozone vers les hautes latitudes, où il s'accumule (figure 4).

Figure 3 - La concentration totale en ozone (intégrée sur toute la verticale) en unités Dobson (tiré de London, 1980). Une unité Dobson vaut 2,69 $\times 10^{16}$ molécules d'ozone par $\mathrm{cm}^{2}$. Elle représenterait $0,01 \mathrm{~mm}$ d'ozone si la colonne d'ai était ramenée à la température de $0{ }^{\circ} \mathrm{C}$ et à la pression standard de surface. Cette distribution est celle d'une atmosphère non perturbée, c'est-à-dire hors de la formation du " trou d'ozone ». Dans l'hémisphère sud, la source principale d'ozone de la troposphère est la stratosphère ; dans I'hémisphère nord, elle résulte surtout de réactions chimiques liées aux activités humaines.

(1) Le ppmv (ou ppm) représente une concentration relative de $10^{-6}$ (une partie par million en volume, soit une molécule d'ozone pour $10^{6}$ molécules d'air). De même, le ppbv (ou ppb) représente une concentration relative de $10^{-9} \mathrm{et} \mathrm{le} \mathrm{pptv}$ (ou ppt) une concentration relative de $10^{-12}$. 
FORMATION ET DESTRUCTION D'OZONE DANS LA STRATOSPHÈRE Chapman et Nicolet

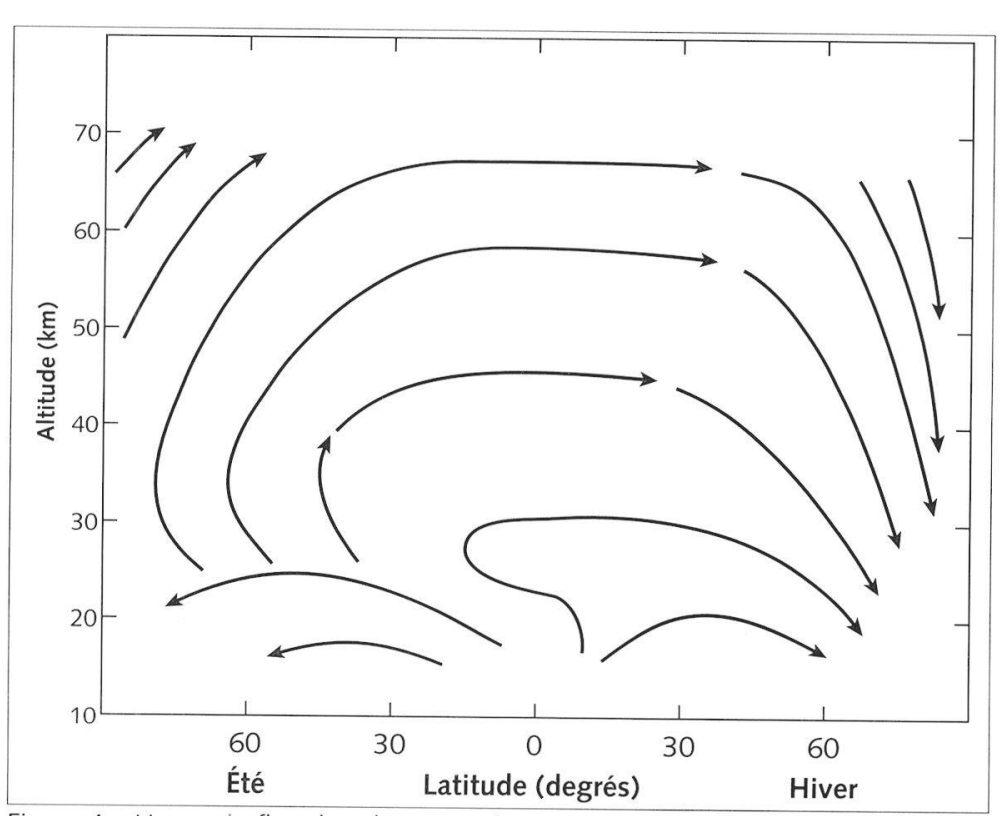

Figure 4 - Lignes de flux dans la stratosphère. L'origine de cette circulation est liée au chauffage inégal de la stratosphère et à une circulation de Hadley qui en résulte (tiré de Dunkerton, 1978). 1930 :
Dans la stratosphère, l'ozone est produit par la photodissociation de la molécule d'oxygène par le rayonnement ultraviolet du Soleil :

$\left.\begin{array}{l}\mathrm{O}_{2}+\mathrm{h} v(\lambda \leqslant 242 \mathrm{~nm}) \longrightarrow \mathrm{O}+\mathrm{O} \\ 2\left[\mathrm{O}+\mathrm{O}_{2}+\mathrm{M} \longrightarrow \mathrm{O}_{3}+\mathrm{M}\right] \\ \mathrm{Net}: 3 \mathrm{O}_{2}+\mathrm{h} v \longrightarrow 2 \mathrm{O}_{3}\end{array}\right\}$

Mais l'ozone est aussi reconverti en oxygène selon un mécanisme de recombinaison proposé pour la première fois par le Britannique Sydney Chapman en

$\left.\begin{array}{l}\mathrm{O}_{3}+\mathrm{h} v(\lambda \leqslant 1140 \mathrm{~nm}) \longrightarrow \mathrm{O}+\mathrm{O}_{2} \\ \mathrm{O}+\mathrm{O}_{3} \longrightarrow 2 \mathrm{O}_{2} \\ \mathrm{Net}: 2 \mathrm{O}_{3}+\mathrm{h} v \longrightarrow 3 \mathrm{O}_{2}\end{array}\right\}$

Le maximum d'ozone dans la stratosphère s'explique donc par le fait que :

- près du sol, le rayonnement UV indispensable pour sa formation est insuffisant ;

- au-dessus de la stratosphère, il n'existe pas assez d'oxygène.

Toutefois, les premières mesures montrèrent que le modèle de Chapman prévoyait beaucoup trop d'ozone et qu'il devait donc exister d'autres réactions contribuant à sa destruction. C'est ainsi que le Belge Marcel Nicolet (Bates et Nicolet, 1950 ; Nicolet, 1970) fut un pionnier de l'explication du rôle important joué par les radicaux hydroxyles $\mathrm{OH}$ et $\mathrm{HO}_{2}$ dans les réactions de destruction de l'ozone (voir réactions 3). Le radical $\mathrm{OH}$ est formé dans la stratosphère à partir de la vapeur d'eau, $\mathrm{H}_{2} \mathrm{O}$, du méthane, $\mathrm{CH}_{4}$, ou de l'hydrogène, $\mathrm{H}_{2}$, et cela, à partir d'un atome d'oxygène excité $\mathrm{O}\left({ }^{1} \mathrm{D}\right)$ produit par le rayonnement ultraviolet du Soleil :

$$
\begin{aligned}
& \mathrm{O}_{3}+\mathrm{h} v(\lambda \leqslant 310 \mathrm{~nm}) \rightarrow \mathrm{O}\left({ }^{1} \mathrm{D}\right)+\mathrm{O}_{2} \\
& \mathrm{H}_{2} \mathrm{O}+\mathrm{O}\left({ }^{1} \mathrm{D}\right) \longrightarrow 2 \mathrm{OH}^{\cdot}
\end{aligned}
$$

\section{Crutzen et les oxydes d'azote}

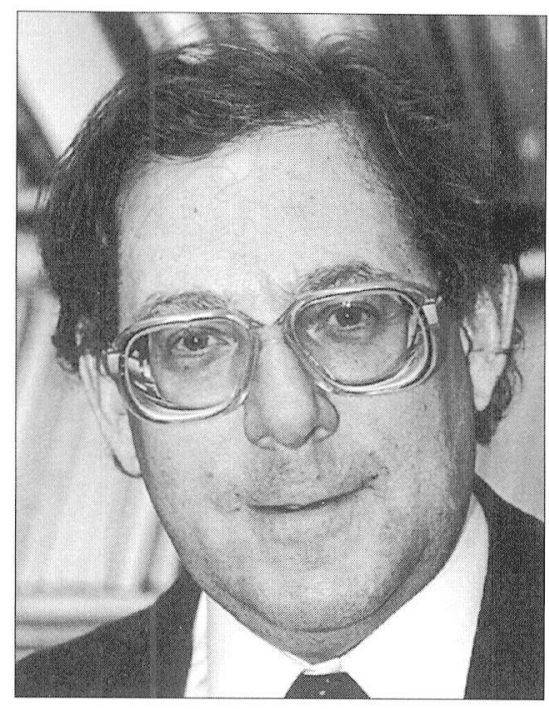

Dans les années soixante-dix, les chercheurs montrèrent, en effet, comment certains constituants mineurs de l'atmosphère pouvaient détruire l'ozone stratosphérique par des réactions catalytiques telles que :

$\left.\begin{array}{l}\mathrm{X}^{\cdot}+\mathrm{O}_{3} \longrightarrow \mathrm{XO}^{\circ}+\mathrm{O}_{2} \\ \mathrm{O}_{3}+\mathrm{hv} \longrightarrow \mathrm{O}+\mathrm{O}_{2} \\ \mathrm{O}+\mathrm{XO}^{\cdot} \longrightarrow \mathrm{X}+\mathrm{O}_{2} \\ \mathrm{Net}: 2 \mathrm{O}_{3}+\mathrm{hv} \longrightarrow 3 \mathrm{O}_{2}\end{array}\right\}$

où $\mathrm{X}^{*}$ et $\mathrm{XO}^{*}$ sont les radicaux qui catalysent la conversion de $\mathrm{O}_{3}$ vers $\mathrm{O}_{2}$.

En 1970, Paul Crutzen (Crutzen, 1970) établit que les plus importants de ces catalyseurs étaient les oxydes d'azote (cycle NO). Ces NO sont liés aux émissions de $\mathrm{N}_{2} \mathrm{O}$ libérées par les processus microbiologiques des sols qui ont reçu de grandes quantités d'engrais. $\mathrm{Ce} \mathrm{N}_{2} \mathrm{O}$ est stable dans la troposphère, mais il est décomposé dans la stratosphère, en particulier par l'oxygène atomique excité produit par le rayonnement UV du Soleil :

$\mathrm{N}_{2} \mathrm{O}+\mathrm{O}\left({ }^{1} \mathrm{D}\right) \rightarrow 2 \mathrm{NO}$

NO peut également être injecté directement dans la stratosphère :

- par les avions (voir le problème des transports supersoniques aux États-Unis, avec Harold Johnston en 1971),

- par les explosions nucléaires à l'air libre,

- par les protons solaires.

Photo d'archive de 1984 montrant le Néerlandais Paul Crutzen, directeur de l'Institut Max Planck, à Mayence, en Allemagne. (C) AFP Photo, W. Eilmes) 


\section{Le chlore et Stolarski-Cicerone}

\section{Les CFC et Molina-Rowland} Les CFC

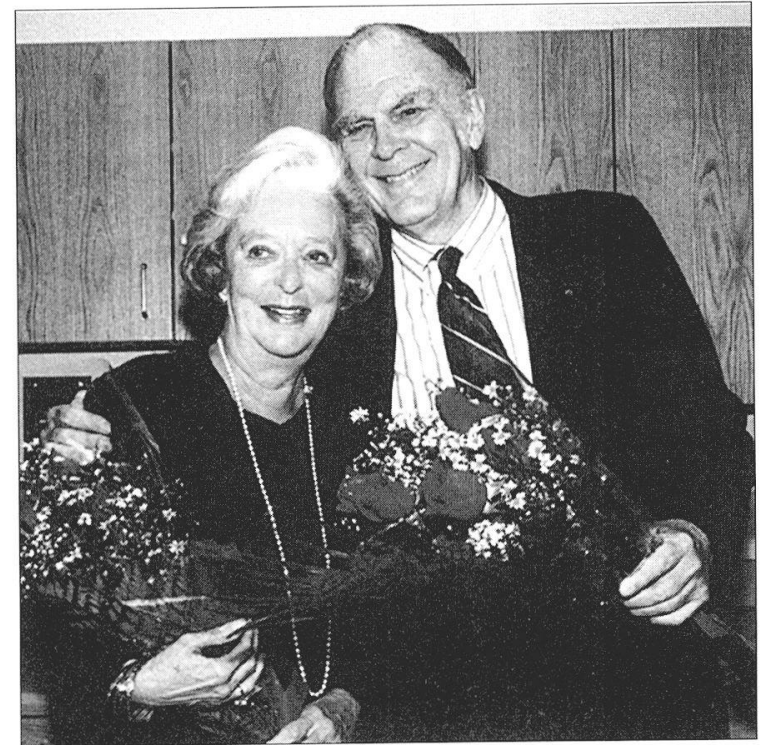
radical $\mathrm{Cl}$.
Dans la troposphère, NO est aussi produit par l'activité microbienne des sols, par la combustion des fiouls fossiles, par les feux de forêts et les éclairs ; mais il y est transformé en $\mathrm{HNO}_{3}$ qui précipite immédiatement.

C'est en 1974 que Richard Stolarski et Ralph Cicerone (Stolarski et Cicerone, 1974), alors à l'université de Michigan, proposèrent le chlore comme radical catalyseur dans les réactions 3. Le $\mathrm{ClO}^{\circ}$ formé par la réaction 3.1 est chimiquement réactif et réagit avec un atome d'oxygène pour relibérer un atome de chlore et fournir une nouvelle molécule d'oxygène (réaction 3.3). L'oxygène atomique est particulièrement abondant dans la haute stratosphère, entre 30 et $50 \mathrm{~km}$ d'altitude, et la réaction 3.3 y prend une à deux minutes sous l'éclairement solaire. L'atome de chlore sera quant à lui fourni par la photodissociation des chlorofluorocarbures (CFC), comme on va le voir à la section suivante.

Ces deux réactions, 3.1 et 3.3 , se reproduisent indéfiniment. Cette chaîne de réactions, appelée cycle $\mathrm{ClO}_{x}$, ne sera interrompue que par l'apparition d'une réaction chimique compétitive qui conduit à la formation d'une molécule réservoir, comme l'acide chlorhydrique $\mathrm{HCl}$.

La même année, Mario Molina et Sherwood Rowland (1974) montrèrent que la photodissociation des chlorofluorocarbures produisait de façon efficace le

Il s'agit principalement des CFC-11 $\left(\mathrm{CFCl}_{3}\right)$ et CFC-12 $\left(\mathrm{CF}_{2} \mathrm{Cl}_{2}\right)$, dont les concentrations dans l'air en surface en 1990 étaient de 280 et 484 pptv respectivement, avec un accroissement annuel de $4 \%$ environ (figure 5). Leur durée de vie est de l'ordre de la centaine d'années. Suite à la signature du Protocole de Montréal en 1987, la production annuelle semble se stabiliser, voire décroître. En 1975, elle était de $310 \mathrm{kt}$ (kilotonnes) et $430 \mathrm{kt}$ de CFC respectivement (figure 6). Les principaux producteurs sont les États-Unis d'Amérique et 1'Union européenne (environ $35 \%$ chacun en 1980). Ces CFC sont utilisés principalement pour les aérosols propulseurs (50-60\%), pour les réfrigérants $(\sim 20 \%)$ et pour les mousses $(\sim 15 \%)$, mais ces proportions diffèrent largement d'une région du monde à l'autre et, avec la quantité produite, évoluent dans le temps. Les halons (bromes allogènes), bien qu'en concentration cent fois moindre, ont aussi des durées de vie de la centaine d'années. De plus, molécule pour molécule, les halons détruisent plus d'ozone, car les radicaux $\mathrm{Br}^{\circ}$ et $\mathrm{BrO}^{*}$ sont moins convertis en $\mathrm{HBr}$, une forme acide moins réactive que dans le cas du $\mathrm{Cl}$.

Les mesures liées au Protocole de Montréal tendent à remplacer de plus en plus ces CFC par des hydrochlorofluorocarbures (HCFC) et des hydrofluorocarbures (HFC). Ceux-ci sont en effet détruits par le radical $\mathrm{OH}^{*}$ dans la troposphère et ont donc des durées de vie beaucoup plus courtes.
F. Sherwood Rowland et sa femme, Joan, après l'annonce du prix Nobel, le 11 octobre 1995, à Irvine, en Californie. (C) AFP Photo, H. Garb)
Figure 5 - Concentration en $\mathrm{CFC}-11\left(\mathrm{CFCl}_{3}\right)$ entre 1977 et 1993 à divers sites d'observation. Le forçage radiatif qui en résulte est repris sur l'échelle de droite (tiré de IPCC, 1994, p. 21).

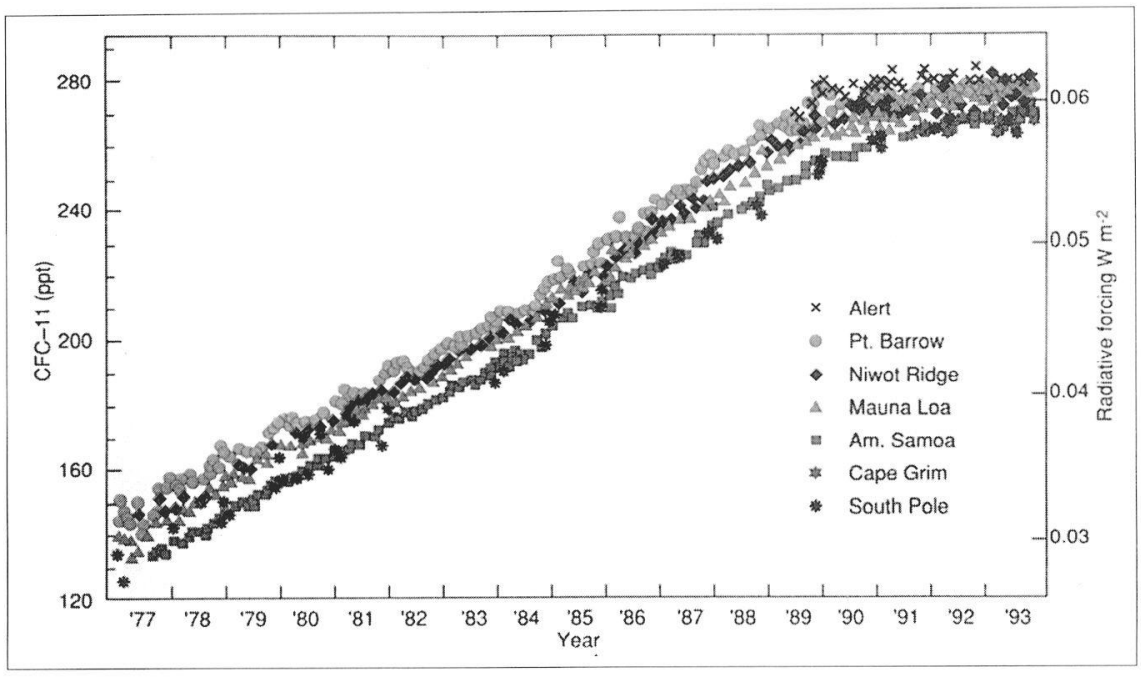


Production de chlore

Ces $\mathrm{CFC}$ ne sont pas détruits dans la troposphère et les quantités émises, $800 \mathrm{kt}$ de chlore par an, finissent par atteindre la stratosphère (une molécule met en moyenne à peu près dix ans). $160 \mathrm{kt}$ des $240 \mathrm{kt}$ qui atteignent la stratosphère fournissent le $\mathrm{Cl}$ inorganique et $80 \mathrm{kt} \mathrm{s}$ 'y accumulent (figure 7). Même si les flux naturels dans le cycle du chlore sont 2000 à 3000 fois supérieurs, il faut insister sur le fait qu'ils se font dans les deux sens, contrairement à ceux d'origine industrielle, car solubilité, réactivité et durée de vie sont différentes.

Au-dessus de 20 à $25 \mathrm{~km}$, le rayonnement solaire est suffisamment énergétique pour les dissocier et produire du chlore atomique :

$$
\left.\begin{array}{l}
\mathrm{CFCl}_{3}+\mathrm{h} v(\lambda \leqslant 260 \mathrm{~nm}) \stackrel{\mathrm{nO}_{2}}{\longrightarrow} \mathrm{CO}_{2}+\mathrm{HF}+3\left(\mathrm{Cl}^{-} \text {ou } \mathrm{ClO}{ }^{\circ}\right) \\
\mathrm{CF}_{2} \mathrm{Cl}_{2}+\mathrm{h} v \longrightarrow \mathrm{Cl}^{*}+\mathrm{CF}_{2} \mathrm{Cl}
\end{array}\right\}
$$

Par les réactions 3, le chlore détruit alors l'ozone de manière plus efficace encore que NO. Une autre chaîne de destruction de l'ozone par le chlore a aussi été mise en évidence par Mario et Luisa Molina au Massachusetts Institute of Technology :

$$
\left.\begin{array}{l}
2\left[\mathrm{Cl}^{\circ}+\mathrm{O}_{3} \longrightarrow \mathrm{ClO}^{*}+\mathrm{O}_{2}\right] \\
\mathrm{ClO}^{*}+\mathrm{ClO}^{\circ}+\mathrm{M} \longrightarrow \mathrm{Cl}_{2} \mathrm{O}_{2}+\mathrm{M} \\
\mathrm{Cl}_{2} \mathrm{O}_{2}+\mathrm{h} v(\lambda \leqslant 400 \mathrm{~nm}) \longrightarrow 2 \mathrm{Cl}^{*}+\mathrm{O}_{2} \\
\mathrm{Net}: 2 \mathrm{O}_{3}+\mathrm{h} v \longrightarrow 3 \mathrm{O}_{2}
\end{array}\right\}
$$
sont mesurées à Ragged Point, Barbades (d'après CDIAC, 1993).

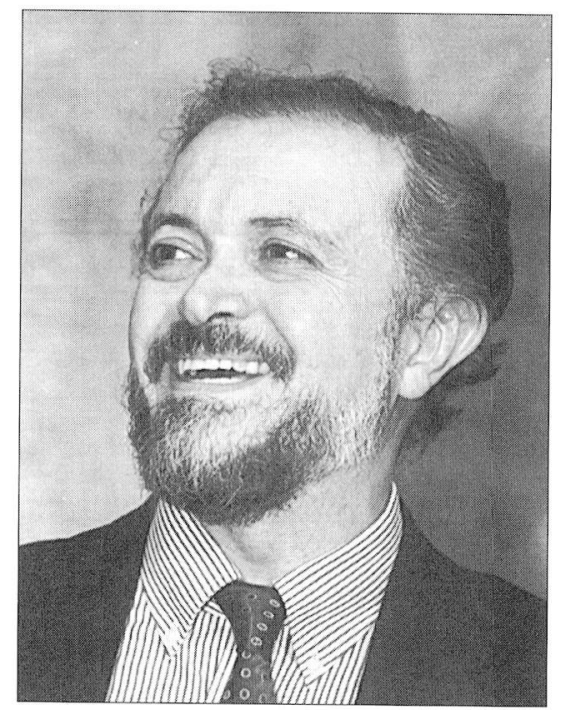

Mario Molina, au cours d'une conférence de presse au Massachusetts Institute of Technology, aux États-Unis, après l'annonce du prix Nobel, le 11 octobre 1995. (C) AFP Photo, S. Cahill)

Neutralisation des catalyseurs
Le chlore peut aussi être injecté dans la stratosphère par les éruptions volcaniques et par le transfert de $\mathrm{CH}_{3} \mathrm{Cl}$ produit par les algues marines. Mais ces sources naturelles sont responsables d'une concentration en chlore dans la stratosphère de 0,6 ppbv, alors qu'elle est de 3 ppbv en ce qui concerne les CFC et autres composés chlorés organiques d'origine industrielle.

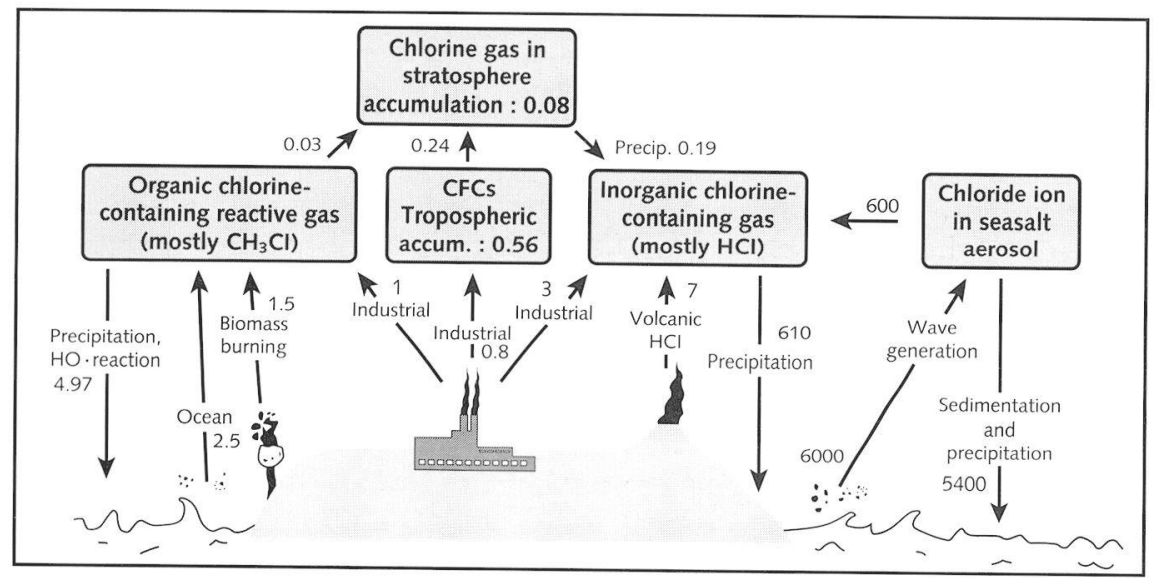

Figure 7 - Cycle du chlore dans l'atmosphère. Les unités sont en mégatonnes de chlore par an (tiré Graedel et Crutzen, 1993). Reproduit avec la permission des auteurs, Atmospheric Change. (C), 1993 by W. H. Freeman and Co.

Il existe des réactions entre les catalyseurs eux-mêmes, réactions qui devraient freiner la disparition de l'ozone :

$\left.\begin{array}{l}\mathrm{OH}^{\cdot}+\mathrm{NO}_{2}+\mathrm{M} \longrightarrow \mathrm{HNO}_{3}+\mathrm{M} \\ \mathrm{ClO}+\mathrm{NO}_{2}+\mathrm{M} \longrightarrow \mathrm{ClONO}_{2}+\mathrm{M}\end{array}\right\}$

Toutefois, cette neutralisation n'est que passagère, car les molécules d' $\mathrm{HNO}_{3}$ et $\mathrm{ClONO}_{2}$ sont photodissociées à leur tour :

$\mathrm{HNO}_{3}+\mathrm{hv}(\lambda \leqslant 330 \mathrm{~nm}) \longrightarrow \mathrm{OH}^{\cdot}+\mathrm{NO}_{2}$

$\left.\mathrm{ClONO}_{2}+\mathrm{h} v(\lambda \leqslant 450 \mathrm{~nm}) \longrightarrow \mathrm{ClO}^{\circ}+\mathrm{NO}_{2}\right\}$

Les modèles qui incorporent toutes ces réactions liées aux cycles $\mathrm{N}, \mathrm{H}$ et $\mathrm{Cl}$ montrent que les émissions de CFC conduisent à une destruction importante de l'ozone dans la stratosphère.

Des analyses plus récentes encore ont montré que la destruction de l'ozone stratosphérique pouvait aussi être liée à des réactions chimiques qui se passent sur des particules gelées faites d'acide nitrique et d'eau. 
- $\mathrm{Si} \mathrm{HNO}_{3}$ gèle et précipite, il ne peut plus être décomposé en $\mathrm{NO}_{2}$ qui normalement détruit $\mathrm{ClO}^{\circ}$ (voir réactions 7.1 et 6.2).

- De plus, en présence d'HCl, la réaction suivante produit du chlore moléculaire : $\mathrm{ClONO}_{2}+\mathrm{HCl} \longrightarrow \mathrm{Cl}_{2}+\mathrm{HNO}_{3}$

$\mathrm{Cl}_{2}$ étant alors photodissociée pour donner deux radicaux $\mathrm{Cl}$ :

$\mathrm{Cl}_{2}+\mathrm{hv}(\lambda \leqslant 450 \mathrm{~nm}) \rightarrow 2 \mathrm{Cl}^{\circ}$

La réaction 8 est cependant très lente (et donc négligeable) en phase gazeuse. En présence de cristaux de glace, tels qu'ils existent dans les nuages stratosphériques polaires (PSC en anglais), le nitrate de chlore et $\mathrm{HCl}$ sont adsorbés, la réaction 8 est beaucoup plus rapide et on a :

$\mathrm{ClONO}_{2}+\mathrm{HCl} \stackrel{\text { glace }}{\longrightarrow} \mathrm{Cl}_{2}(\mathrm{gaz})+\mathrm{HNO}_{3}$ (gelé)

Le chlore moléculaire peut donc être formé, mais $\mathrm{NO}_{2}$ ne peut être produit à partir de $\mathrm{HNO}_{3}$ et ne peut donc plus protéger (voir réaction 6.2) l'ozone de sa destruction par les radicaux $\mathrm{Cl}^{*}$ et $\mathrm{ClO}^{*}$.

L'ensemble de ces réactions catalytiques permet ainsi une destruction importante de l'ozone stratosphérique : Rowland (1990) estime qu'un atome de chlore permet de détruire 100000 molécules d'ozone. Les échanges entre les chaînes de réactions et les réservoirs temporaires peuvent se faire de 50 à 200 fois, chaque chaîne pouvant se boucler un millier de fois.

TROU D'OZONE DANS L'ANTARCTIQUE ET DANS L'ARCTIQUE

Figure 8 - (a) Distribution verticale de l'ozone au-dessus de McMurdo, Antarctique, en 1987

(tiré de Rowland, 1990).

(b) Comparaison entre le profil d'ozone avant le printemps (2 septembre 1994) et le profil où la concentration atteint son minimum

(12 octobre 1993 et 5 octobre 1994) au pôle Sud. Les différences dans la zone de 10 à $12 \mathrm{~km}$ sont dues aux altitudes différentes de la tropopause (tiré de Hofmann et al., 1995).
Pendant la longue nuit polaire, l'absence de soleil et la diminution des échanges atmosphériques avec les latitudes plus basses favorisent l'apparition de températures très basses et de nuages stratosphériques polaires. Comme le montre la figure 3 , ces conditions sont favorables à des concentrations faibles en ozone aux pôles où un minimum apparaît dès le début de l'hiver (septembre octobre au pôle nord et mars au pôle sud). Au printemps, en avril au pôle nord et en novembre au pôle sud, on y constate au contraire un maximum. Depuis le début des années quatre-vingt, avec la présence du chlore actif, on s'aperçoit que dans l'Antarctique, en septembre, dès le retour du rayonnement solaire, les mécanismes photochimiques conduisent à la destruction de l'ozone. En réalité, lorsque l'on parle du «trou d'ozone », on se réfère en fait à l'accélération de la diminution de la concentration en ozone dans la stratosphère antarctique ou arctique. C'est en 1985 que l'Anglais Joseph Farman et ses collègues du British Antarctic Survey (Farman et al., 1985) observent une réduction drastique de la couche d'ozone dans la stratosphère antarctique au printemps (octobre). Cette réduction est surtout spectaculaire vers les $15 \mathrm{~km}$ d'altitude (figures $8 \mathrm{a}$ et $8 \mathrm{~b}$ ), mais est tout aussi visible dans la colonne atmosphérique (figures 9a et 9b).
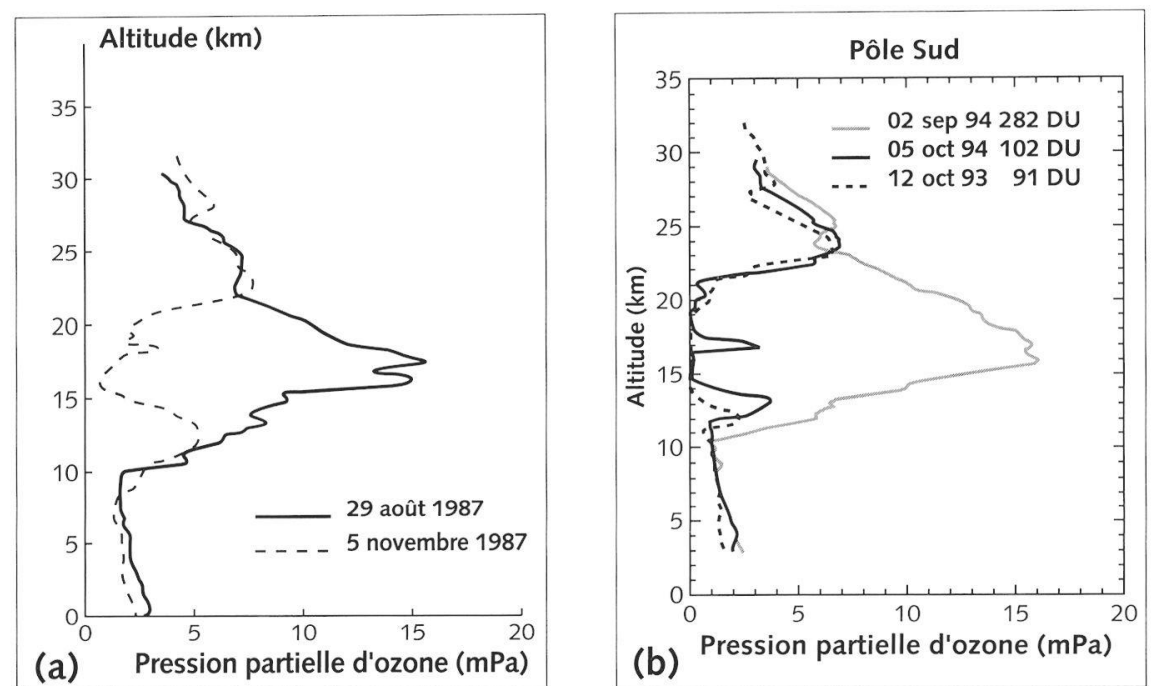
Figure 9 - (a) Épaisseur de la couche d'ozone (moyenne mensuelle en octobre) au-dessus de Halley Bay, Antarctique, en unités Dobson. On notera la forte diminution depuis 1970 (d'après Farman et al., 1985 ; Graedel et Crutzen, 1993).

(b) Contenu en ozone dans toute la colonne atmosphérique (figure supérieure) et dans la tranche de 12 à $20 \mathrm{~km}$ (figure inférieure), mesuré à partir de ballons au pôle Sud durant la période du " trou d'ozone " au printemps, de 1992 à 1994, en unités Dobson. La moyenne 1986-1991 est représentée par la courbe pleine et les courbes situées à plus ou moins un écart type sont tiretées (tiré de Hofmann et al., 1995).

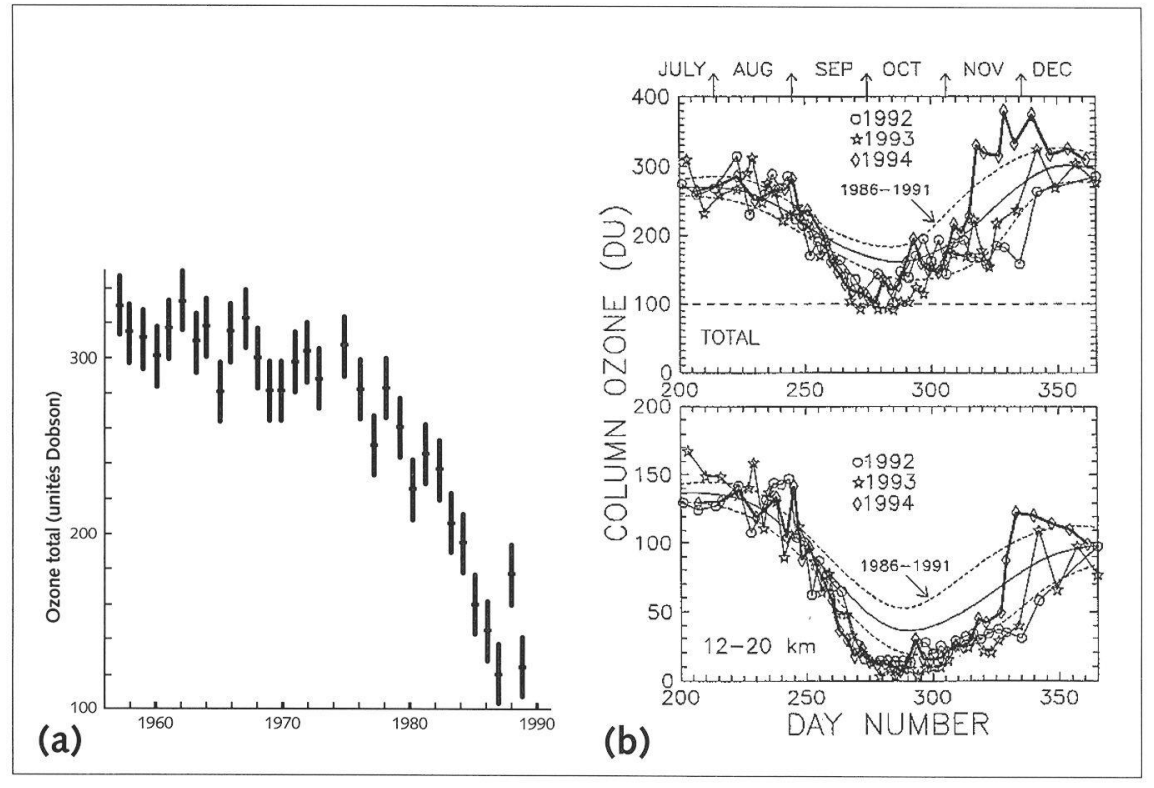

En septembre 1995, le trou d'ozone antarctique s'étendait sur 22 millions de $\mathrm{km}^{2}$ avec, en son centre, un contenu intégré de 130 Dobson, ce qui représente une réduction de 55 à $60 \%$ de la valeur «normale ». Au cours des dernières années, la valeur minimale est descendue en dessous des 100 Dobson et, à plusieurs reprises, l'ozone a totalement disparu vers les $15 \mathrm{~km}$.

L'observation a aussi pu mettre en évidence l'importance du chlore comme le montre la figure 10 : brusquement, la concentration en $\mathrm{ClO}^{\circ}$ augmente en même temps que la concentration en ozone diminue. De plus, la chimie en phase hétérogène explique en partie la différence de comportement entre 1'Antarctique et l'Arctique. En hiver, vu l'altitude du continent antarctique, la température y est très basse dans la stratosphère $\left(-80^{\circ} \mathrm{C}\right.$ et au-delà $)$. De plus, le tourbillon circumpolaire antarctique y isole 1'atmosphère du reste de la circulation générale. Cela rend le milieu confiné et permet la formation de particules solides $\left(\mathrm{HNO}_{3}\right.$ gelé et glace sur lesquels les composants qui séquestrent le chlore se condensent) ainsi que la rétention de l'air riche en chlore pendant des mois.

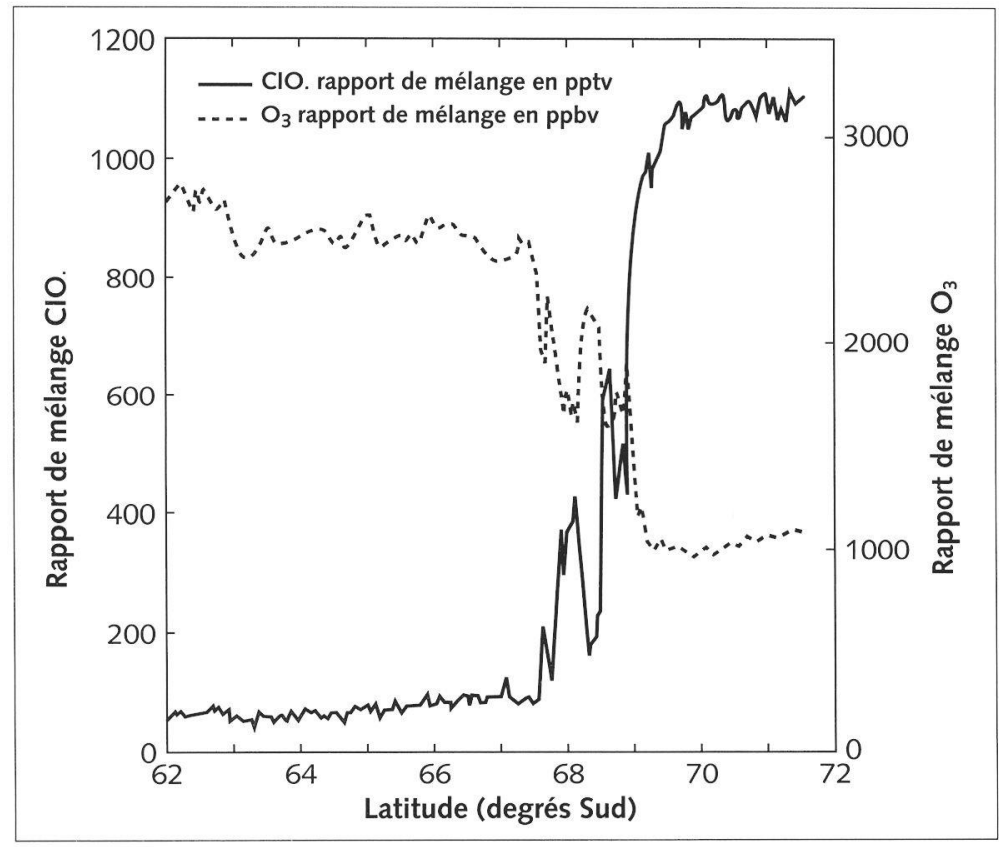

Figure 10 - Monoxyde de chlore et ozone mesurés à partir des instruments sur le vol ER-2, le 16 septembre 1987, entre $53^{\circ} \mathrm{S}$ (Punta Arenas, Chili) et $72^{\circ} \mathrm{S}$ en Antarctique (tiré de Rowland, 1990).
Alors que les plus faibles concentrations jamais enregistrées avaient été relevées, en septembre 1994, au-dessus de l'Antarctique, en présence d'un immense trou d'ozone d'environ 24 millions de $\mathrm{km}^{2}$ (Bojkov, 1995), les données recueillies en temps quasi réel dans le cadre du Système mondial d'observation de l'ozone de l'Organisation météorologique mondiale et de la Second European Stratospheric Arctic and Midlatitude Experiment (Sesame) indiquent que des concentrations en ozone exceptionnellement basses ont persisté durant la plus grande partie de janvier et pendant la première moitié du mois de février 1995, au-dessus des latitudes septentrionales moyennes, notamment de la Sibérie, s'étendant jusqu'en Europe occidentale. Le déficit en ozone a été de $25 \%$ par rapport aux valeurs moyennes à long terme au-dessus de la Sibérie durant la seconde moitié du mois de janvier, et il s'est maintenu jusqu'au 25 février. Pendant plusieurs jours, ce déficit a dépassé $35 \%$, alors que les valeurs d'ozone total enregistrées étaient de 1'ordre de 250 à 270 Dobson (les valeurs moyennes à long terme étant d'environ 440 Dobson). Durant la même période, le déficit moyen au-dessus de l'Europe occidentale et 
centrale, comme au-dessus de la partie occidentale de l'Amérique du Nord, était de 10 à $12 \%$ (soit à peu près à la limite statistique de ce que l'on considère comme une variation normale) ; cependant, certaines semaines, le déficit en ozone enregistré au-dessus de 1'Europe a dépassé les $20 \%$. Localement, des augmentations importantes du rayonnement UV-B ont été mesurées pendant les périodes de basse concentration en ozone. Les températures de la basse stratosphère, au-dessus de la Sibérie et de la partie orientale de l'Europe, étaient de 10 à $12{ }^{\circ} \mathrm{C}$ inférieures à la normale, ce qui a facilité la destruction chimique de l'ozone par des composés chlorés et bromés d'origine anthropique. Ces températures extrêmement basses (record des vingt-neuf dernières années) sont probablement dues à la variabilité naturelle du climat et ne devraient donc pas nécessairement se reproduire chaque année.

\section{RÉDUCTION DE L'OZONE SUR TOUTE LA TERRE}

\section{LA COUCHE D'OZONE ET LE CLIMAT}

Bien que la réduction soit la plus spectaculaire au-dessus de l'Antarctique, on assiste à un phénomène mondial tel que l'on peut le voir sur la figure 11. La diminution du contenu intégré de l'ozone varie de $3 \%$ par an dans l'Antarctique à 1,5\% par an dans 1'Arctique lors de leur printemps respectif. La réduction s'étend jusqu'aux latitudes de $20^{\circ}$. Entre $35^{\circ} \mathrm{S}$ et $90^{\circ} \mathrm{S}$, la diminution est aussi spectaculaire et atteint $10 \%$ par rapport aux années antérieures à 1980 (figure 12).

Le «trou d'ozone » a aussi des connexions avec le problème de l'intensification de l'effet de serre (Berger, 1992). L'ozone et les chlorofluorocarbures sont des gaz à effet de serre, tout comme le dioxyde de carbone et le méthane. Molécule pour molécule, le CFC-11 est 15800 fois plus efficace que le $\mathrm{CO}_{2}$ pour piéger l'énergie infrarouge et intensifier ainsi l'effet de serre naturel. En fait, une partie de l'intensification de l'effet de serre due aux CFC compense l'effet négatif induit par la diminution de l'ozone stratosphérique (IPCC, 1994). Quant à l'ozone de la troposphère, les modèles montrent que le climat est particulièrement sensible aux changements de sa concentration dans les basses couches atmosphériques. Là, l'ozone a considérablement augmenté au cours des cent dernières années, principalement à cause des émissions d'oxyde d'azote $\mathrm{NO}_{x}\left(\mathrm{NO}+\mathrm{NO}_{2}\right)$, de monoxyde de carbone, $\mathrm{CO}$, de méthane, $\mathrm{CH}_{4}$, et autres hydrocarbures (tels que l'éthylène, le butane, etc.). Ces émissions proviennent principalement des véhicules de transport, des industries et de la combustion de la biomasse sous les tropiques. P. Crutzen a joué un rôle clé dans l'établissement des cycles d'oxydation de $\mathrm{CO}$ et $\mathrm{CH}_{4}$ pour produire de l'ozone dans la basse troposphère en présence de $\mathrm{NO}_{x}$ comme catalyseurs (Graedel et Crutzen, 1993). Cet ozone dans les basses couches est lui-même source de problèmes importants pour l'environnement en raison des dommages qu'il cause aux végétaux et à la santé humaine (voir l'article de G. Mégie dans ce même numéro de La Météorologie).

On estime actuellement que l'augmentation des CFC et de l'ozone dans la troposphère est responsable respectivement de $15 \%$ et $8 \%$ environ du réchauffement climatique calculé par les modèles. D'autre part, la diminution de l'ozone stratosphérique a pour effet de faire baisser localement de quelques degrés les températures de la moyenne stratosphère, mais participe aussi à réduire le réchauffement climatique mondial de quelques pour cents.

Figure 11 - Écart (en pourcentage) entre les quantités d'ozone total relevées pendant deux périodes (19641980 et $1984-1993)$. Au sud de $60^{\circ}$ S, l'écart dépasse $15 \%$ entre septembre et novembre, avec un maximum de $35 \%$ en octobre à partir de $80^{\circ} \mathrm{S}$ vers le pôle (tiré de Bojkov, 1995). Cela représente un taux annuel de décroissance de $0,1 \%$ à l'équateur à plus de $3 \%$ au pôle Sud en septembre-octobre ; ces résultats sont comparables à ceux de Stolarski et al. (1991). 


\section{CONSÉQUENCES DE L'ALTÉRATION DE LA COUCHE D'OZONE}

\section{Sur la santé humaine}

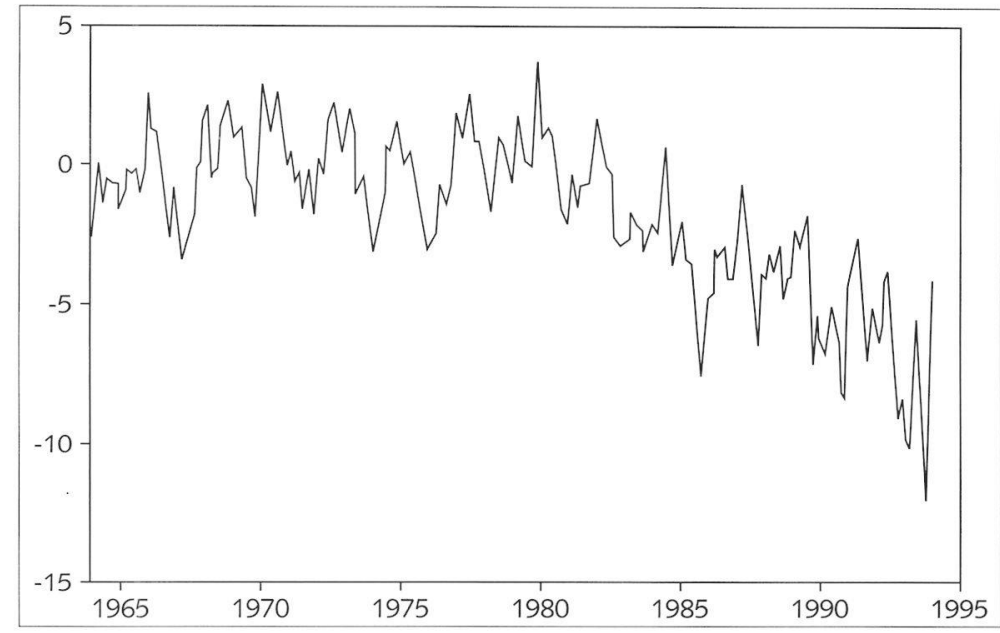

Figure 12 - Écarts par rapport aux contenus d'ozone de la période 1964-1980, aux latitudes comprises entre $35^{\circ} \mathrm{S}$ et $90^{\circ} \mathrm{S}$ (en pourcentage). La diminution au cours des vingt dernières années est spectaculaire et atteint $10 \%$ (tiré de Bojkov, 1995). et Jensen, 1995). rieures de l'œil.
Parmi les produits de substitution aux CFC, on trouve les hydrochlorofluorocarbures. Leur durée de vie plus courte leur donne un potentiel destructeur d'ozone beaucoup plus faible, mais par contre, ils ont un potentiel de réchauffement qui demeure important. Brûler dans le présent ou cuire dans l'avenir !

La diminution du contenu intégré de l'ozone dans la stratosphère conduit à une augmentation du rayonnement ultraviolet au sol, particulièrement dans la partie $\mathrm{B}$ du spectre. On estime que toute réduction de $1 \%$ de la couche d'ozone augmente de $2 \%$ la dose d'UV-B. Ce rayonnement est particulièrement néfaste pour les organismes vivants, car il est absorbé par des molécules vitales, telles que l'ADN, et les endommage. Des études de plus en plus nombreuses confirment l'importance de ces UV-B dans l'apparition de cancers de la peau et d'affections oculaires, dans l'affaiblissement du système immunitaire et dans les dommages créés aux plantes tant terrestres que marines (Van der Leun et al., 1994). Il faut noter, toutefois, que pour avoir un impact réel, l'augmentation de ce rayonnement UV-B doit être supérieure à la variabilité naturelle induite par d'autres facteurs, tels que la transmission atmosphérique. Une étude récente montre que c'est déjà le cas en certains endroits, pour les latitudes supérieures à $30^{\circ}$ et par ciel clair ; de toute façon, cela semble inévitable dans l'avenir (Lubin

Le rayonnement UV-B est fortement absorbé par la peau et les couches exté-

L'œil est moins protégé naturellement que la peau, mais est fort heureusement moins exposé. Il n'empêche que les effets à long terme des UV-B sur l'œil peuvent conduire à une diminution sérieuse de l'acuité visuelle, et même à la perte de la vue. On estime, par exemple, qu'une diminution de la couche d'ozone de 6 à $7 \%$ en été pourrait conduire à une augmentation de $3 \%$ environ du nombre de cataractes au début du siècle prochain.

Même si notre peau a développé ses mécanismes d'autodéfense (épaississement et pigmentation), l'exposition prolongée aux UV-B peut conduire à une tumeur ou à un cancer. Les cancers non malins de la peau sont les plus fréquemment dépistés. Toute augmentation de $1 \%$ du rayonnement UV-B conduirait à une augmentation de 1 à $3 \%$ du nombre de ces carcinomes, selon le type.

Aux États-Unis et en Allemagne, on estime que la diminution de $7 \%$ de l'ozone prévue pour la fin de ce siècle conduirait à une augmentation du nombre de ces cancers de $20 \%$ environ, ce qui représente respectivement 100000 et 20000 cas de plus par an. Les personnes les plus sensibles sont celles aux cheveux roux et celles à la peau blanche. Le risque augmente lors d'une exposition prolongée au soleil des latitudes plus basses, principalement pour les personnes qui viennent des régions tempérées où la nébulosité est plus importante. Les cancers des cellules pigmentaires, connus sous le nom de mélanomes, sont eux mortels, mais fort heureusement moins nombreux (25 fois moins environ que les carcinomes). Toutefois, l'impact de la diminution d'ozone dans la stratosphère est difficile à mettre en évidence, vu le manque crucial de statistiques dans ces matières.

Des expositions continues à une dose accrue d'UV-B, qui pourrait survenir à la suite d'une diminution prolongée de l'ozone stratosphérique, affecterait aussi le système immunitaire, ayant pour conséquence d'augmenter le nombre d'infections et de morts. 
Sur les plantes

\section{Sur les écosystèmes marins}

Les espèces de plantes peuvent réagir de multiples façons à l'augmentation du rayonnement UV-B. Certaines voient leur croissance sérieusement altérée, d'autres variétés peuvent s'adapter assez vite, certaines pourraient même croître plus rapidement. Les autres attaques auxquelles les plantes sont soumises peuvent aussi masquer l'influence directe des UV-B. Sur 200 plantes testées, la moitié a subi une influence significative, telle qu'une diminution de la surface foliaire, des racines et du taux de photosynthèse. Les céréales semblent être particulièrement sensibles.

Les mécanismes de défense des écosystèmes marins sont ceux qui correspondent à des profondeurs marines où le rayonnement UV-B est filtré, mais où les plus grandes longueurs d'onde sont toujours présentes pour assurer la photosynthèse. Un affaiblissement du bouclier protecteur d'ozone se solderait donc par un dommage direct dû à la pénétration possible d'UV-B et par un dommage indirect dû à une diminution de l'activité photosynthétique, le plancton se réfugiant à de plus grandes profondeurs. Comme le plancton occupe une place primordiale dans la chaîne alimentaire, toute perturbation qui l'affecte affecte aussi le zooplancton et les poissons.

L'AVENIR Pour que les «trous d'ozone » n'apparaissent plus à l'avenir, la concentration relative de chlore dans l'atmosphère devrait être ramenée en dessous de 2 ppbv. Elle est à 1'heure actuelle de quelque 3,5 ppbv et, au printemps, dans la stratosphère antarctique éclairée par le Soleil, l'ozone est détruit à un rythme de $2 \%$ par jour environ. Si la teneur en chlore devait atteindre 6 ppbv, l'ozone serait détruit quatre fois plus vite qu'aujourd'hui, c'est-à-dire au rythme de 8 à $10 \%$ par jour.

Il est donc essentiel de supprimer la production de CFC, halons et autres substances destructrices de l'ozone. Cela doit se faire le plus rapidement possible, en tout cas en ne dépassant pas l'échéance de l'an 2000, comme il a été convenu au cours de la Conférence de Londres, en juin 1990, par les parties du Protocole de Montréal (signé en 1987). En principe, $85 \%$ des CFC devraient être bannis d'ici 1997, avec quelque délai pour les pays en voie de développement. Comme la durée de vie de ces gaz qui détruisent la couche d'ozone est relativement longue, on doit s'attendre à une poursuite de la diminution de la concentration en ozone, dans la stratosphère, non seulement au-dessus de l'Antarctique, mais aussi audessus d'une partie de l'hémisphère nord. Grâce aux mesures prises (et à prendre), la destruction de la couche d'ozone devrait diminuer dès le début du $\mathrm{XXI}^{\mathrm{e}}$ siècle, même s'il faudra probablement attendre plus de cent ans avant que cette couche ne soit totalement rétablie.

En fait, les effets de l'éruption du Pinatubo en 1991 étant pratiquement terminés, on aurait pu s'attendre à ce que le trou d'ozone dans l'Antarctique soit moins important en octobre 1995. Les observations montrent que ce ne fut pas le cas, mais que le record de 1994 ne sera pas battu. Un trou de 22 à 23 millions de $\mathrm{km}^{2}$ est apparu avec, en son centre, des contenus intégrés de 98 Dobson, valeurs équivalentes à celles des dernières années.

Toutefois, les modèles montrent que l'on ne devrait pas s'attendre à un trou plus important au cours des prochaines années et que les mesures du Protocole de Montréal devraient faire apparaître une amélioration au début du XXI ${ }^{\mathrm{e}}$ siècle. Si le trou ne s'agrandit ni ne s'approfondit alors que les concentrations en chlore devraient encore augmenter, c'est parce que la disparition de l'ozone, dans les couches où la température permet aux réactions de destruction d'être efficaces, est déjà totale et que les modèles ne prévoient pas un épaississement de ces couches extrêmement froides $\left(\mathrm{T}<-80{ }^{\circ} \mathrm{C}\right)$. On doit cependant s'attendre à une accélération de la disparition printanière de l'ozone dans la décennie qui vient (communication de M. Schoerberl du Goddard Space Flight Center à Greenbelt, Maryland, États-Unis ; communication de A. Neuendorffer et de D. Hofmann de la National Oceanic and Atmospheric Administration à Silver Spring et à Boulder, États-Unis ; Kerr, 1995).

Qui vivra verra! 
BIBLIOGRAPHIE Bates D. R. et M. Nicolet, 1950 : The photochemistry of atmospheric water vapor. $J$. Geophys. Res., 55, 301.

Berger A., 1992 : Le climat de la Terre, un passé pour quel avenir ? De Boeck Université, Bruxelles, 479 p.

Bojkov R. D., 1995 : Évaluation internationale de l'état de la couche d'ozone 1994. Bull. OMM, 44, 1, 46-54.

CDIAC, 1993 : Trends'93. Carbon Dioxide Information Analysis Center, Oak Ridge National Laboratory, États-Unis.

Crutzen P. J., 1970 : The influence of nitrogen oxydes on the atmospheric ozone content. Quart. J. Roy. Meteor. Soc., 96, 320-325.

Dunkerton T., 1978 : On the mean meridional mass motions of the stratosphere and mesosphere. J. Atmos. Sci., 35, 2325-2333.

Farman J. C., B. G. Gardiner et J. D. Shanklin, 1985 : Large losses of total ozone in Antarctica reveals seasonal $\mathrm{ClO}_{\mathrm{x}} / \mathrm{NO}_{\mathrm{x}}$ interaction. Nature, 315, 207-210.

Graedel T. E. et P. Crutzen, 1993 : Atmospheric Change, An Earth System Perspective. W. H. Freeman, New York, États-Unis, 446 p.

Hofmann D. J., S. J. Oltmans, B. J. Johnson, J. A. Lathrop, J. M. Harris et H. Vömel, 1995 : Recovery of ozone in the lower stratosphere at the South Pole. Geophys. Res. Lett., 22, 18, 2493-2496.

IPCC, 1994 : Radiative Forcing of Climate Change. Intergovernmental Panel on Climate Change. WMO-UNEP, $28 \mathrm{p}$.

Kerr R. A., 1995 : Ozone hole won't worsen ? Science, 270, 376.

London J., 1980 : Radiative energy sources and sinks in the stratosphere and mesosphere. In : M. Nicolet et A. C. Aiken (Eds), Proceedings of the NATO Advanced study Institute on atmospheric ozone : its variations and human influences. U. S. Dept. of Transportation, Washington DC, États-Unis, 703-721.

Lubin D. et E. H. Jensen, 1995 : Effects of clouds and stratospheric ozone depletion on ultraviolet radiation trends. Nature, 377, 710-713.

Meteorological Office, 1991 : Meteorological Glossary. HMSO, London, 335 p.

Molina M. et F. S. Rowland, 1974 : Stratospheric sink for chlorofluoromethanes: chlorine atom catalyzed destruction of ozone. Nature, 249, 810-814.

Nicolet M., 1970 : Ozone and hydrogen reactions. Ann. Geophys., 26, 531-536.

Rowland F. S., 1990 : Stratospheric ozone depletion by chlorofluorocarbons. Ambio, $19,6-7,281-292$

Stolarski R. S. et R. J. Cicerone, 1974 : Stratospheric chlorine: a possible sink for ozone. Can. J. Chem., 52, 1610-1615.

Stolarski R. S., P. Bloomfield, R. D. McPeters et J. R. Herman, 1991 : Total ozone trends deduced from Nimbus 7 TOMS data. Geophys. Res. Lett., 18, 1015-1018.

Van der Leun J., M. Tevini et X. Tang (Eds), 1994 : Environmental Aspects of Stratospheric Ozone Depletion, Update. United Nations Environmental Programme, Nairobi.

Wofsy S. C., M. B. McElroy et Y. C. Yung, 1975 : The chemistry of atmospheric bromine. Geophys. Res. Lett., 2, 215-218. 